\title{
New approaches to the rapid diagnosis of tuberculosis
}

Rapid confirmation of a diagnosis of tuberculosis is important for starting appropriate treatment early and for avoiding the inappropriate use of complex and potentially toxic drug regimens. Detection of the intact bacillus, either by direct smear or by cuiture, remains the only available way to confmm diegnosis with certalnty. Cutture the organism is too slow to influence initial decisions on treatment but does allow precise taentincation of bacteral species and asscosment of antibiotic sensitivities. The direct smear is easy to perform and is the only readily available rapid diagnostic method, but depends for a positive result on the presence of more than 5000 organisms per millilitre of sample. 'Smear negative aisease is thus a feature of half of all "putmomary tuberculosis infections and is virtually the rule for infection at other sites, where smaller numbers of organisms cause clinical disease (for example, pleura, meninges). A method for rapid confirmation of tuberculosis in the presence of small numbers of organisms would be a great advance in the management of this common condition. To be useful such a test would have to fulfil rigorous criteria, including speed, sensitivity, ease of performance, applicability in all clinical circumstances, ability to distinguish active from old disease and Mycobacterium tuberculosis from other mycobacteria (specificity), and low cost.

Measurement of the bumoral response to various mycobacterial antigens is the technique that has been most extensively assessed as an alternative to the established methods. Such tests have the advantage that the study material (blood) is nearly always readily available and may render invasive investigation unnecessary. The pattern of antibody response in tuberculosis varies, however, between individuals, and most antibody responses are weak and directed predominantly against non-specific mycobacterial antigens. Despite much research a test suitable for routine use has yet to be developed. ${ }^{2}$ Two publications now suggest that there may be light at the end of the serological tunnel. Both studies used monoclonal antibodies in a solid phase antibody competition test and the second also an ELISA to purified antigens. ${ }^{34} \mathrm{With}$ a specificity of $98 \%$ a single monoclonal antibody to a $M$ tuberculosis specific antigen gave positive results in $85 \%$ of cases of smear positive and $70 \%$ of cases of smear negative pulmonary disease and in 70-80\% of cases of extrapulmontry tuberculosis. Such results would have made most biopsy procedures unnecessary, allowed chemotherapy to have been started on average 12.5 days earlier, and prevented treatment in $93 \%$ of those initially treated inappropriately for tuberculosis. ${ }^{3}$ The second of these studies, by Bothamley et al, reported in this issue of Thorax, ${ }^{4}$ confirms the more limited antibody response in smear negative pulmonary disease, but suggests that a battery of monoclonal antibodies, with differing specificities, produces better results than a single monoclonal antibody. It is also suggested that non-tuberculous mycobacterial infection could be distinguished, and that there is a correlation between the pattern of antibody response and disease severity, extent of radiographic disease, bacteriological relapse, and even compliance with treatment. These optimistic results require confirmation, but suggest that serological tests at last may have a role. Problems still to be resolved include the best choice of epitope to which the monoclonal antibody should be directed, the time consuming nature of multiple assays, the area of overlap between antibody titres in patients with disease and in controls, and cost.

An alternative diagnostic approach is to artificially increase the number of bacteria, or markers of those bacteria, present in a given sample. One of the advantages of using the polymerase chain reaction to amplify mycobac- terial DNA is that the specificity of the method depends on the precise DNA sequence chosen for amplification. This technique has been shown to be successful in detecting mycobacteria, with a greater sensitivity than that of routine smear and culture, in sputum, pleural fluid, cerebrospinal fluid, pericardial fluid, pus, urine, and tissue samples. ${ }^{5}$ The sensitivity depends on the number of amplification cycles and can be increased by the amplification of a DNA sequence present in multiple rather than single copies in each genome. de Lassence and colleagues describe such a technique in this issue. ${ }^{6}$ It allowed the detection of as little as one mycobacterial genome per sample of pleural iruia and confirmed a tuberculous cause for the pleural fluid, without any loss of specificity, in most of the 15 patients, whose tuberculosis was confirmed by other means. The extreme sensitivity of their technique could, however, be its downfall, as false positive results, presumably due to contamination, are reported, indicating the need for scrupulous laboratory methods and practice. ${ }^{7}$ The value of the polymerase chain reaction in "dirty" samples, such as sputum, remains to be proved. Although the technique usually requires material from the site of infection, the positive results obtained from blood from patients with tuberculosis who had AIDS suggest that this may not always be necessary. ${ }^{8}$

Serology and the polymerase chain reaction method may be mutually exclusive or complementary, but further evaluation in prospective studies and direct comparison of the two techniques will be required to determine their respective roles. In future it may be possible to tailor the diagnostic test to particular circumstances. In non-industrialised countries, where most mycobacterial infections are tuberculous, a single monoclonal antibody or DNA sequence specific for $M$ tuberculosis could be used whereas in patients with AIDS, for example, where distinction between types of mycobacterial infection is important, antibodies or DNA sequences with differing specificities will be required.

The potential sensitivity of the polymerase chain reaction method could allow this to replace smear examinations if specificity is maintained, especially in "dirty" samples. In non-industrialised countries, however, the simplicity of the sputum smear and its availability at the point of access to medical care are unlikely to be surpassed by such sophisticated techniques, though they may become a useful addition in smear negative cases. Although the results of both the approaches described are exciting, further work is needed to clarify their specificity and sensitivity in different circumstances and to simplify laboratory procedures. Finally, the practicability of their large scale use requires evaluation.

MARK WOODHEAD

Manchester Royal Infirmary, Consultant physician

Manchester M13 9WL

Reprint requests to: Dr M Woodhead

1 Rouillon A, Perdrizet S, Parrot R. Transmission of tubercle bacilli: the effects of chemotherapy. Tubercle 1976;57:275-99.

2 Grange JM. The rapid diagnosis of paucibacillary tuberculosis. Tubercle 1989;70:1-4.

3 Wilkins EGL, Ivanyi J. Potential value of serology for diagnosis of extrapulmonary tuberculosis. Lancet 1990;336:641-4

4 Bothamley GH, Rudd R, Festenstein F, Ivanyi J. Clinical value of the measurement of $M$ tuberculosis-specific antibody in pulmonary tuberculosis. Thorax 1992;47:9-14.

5 Manjunath N, Shankar P, Rajan L, Bharagva A, Saluja S, Shriniwas. Evaluation of a polymerase chain reaction for the diagnosis of tuberculosis. Tubercle 1991;72:21-7.

6 de Lassence A, Lecossier D, Pierre C, Cadranel J, Stern M, Hance AJ. Detection of mycobacterial DNA in pleural fluid from patients with tuberculous pleurisy using the polymerase chain reaction: a comparison of two protocols. Thorax 1992;47:265-9.

7 Shankar P, Manjunath N, Mohan KK, et al Rapid diagnosis of tuberculous meningitis by polymerase chain reaction. Lancet 1991;337:5-7.

8 Brisson-Noel A, Gicquel B, Lecossier D, Levy-Frebault V, Nassif X, Hance AJ. Rapid diagnosis of tuberculosis by amplification of mycobacterial DNA in clinical samples. Lancet 1989;ii:1069-71. 